\title{
Effect of Particle Size on Mixing Degree in Dispensation
}

\author{
Hitoshi Nakamura, ${ }^{a}$ Yoshitsugu Yanagihara, ${ }^{a}$ Hiroko Sekiguchi,${ }^{b}$ Michiteru Ohtani, ${ }^{b}$ \\ Satoru KARIYA, ${ }^{b}$ Katsuyoshi UCHINO, ${ }^{b}$ Hiroshi SuzUKI, ${ }^{a}$ and Tatsuji IGA ${ }^{*, a}$ \\ Department of Pharmacy, The University of Tokyo Hospital, Faculty of Medicine, The University of Tokyo, ${ }^{a}$ \\ 7-3-1 Hongo, Bunkyo-ku, Tokyo 113-8655, Japan and Department of Hospital Pharmacy, Tokyo Postal \\ Service Agency Hospital, b 2-14-23 Fujimi, Chiyoda-ku, Tokyo 102-8798, Japan
}

(Received September 26, 2003; Accepted December 11, 2003)

\begin{abstract}
By using lactose colored with erythrocin, we examined the effect of particle size on mixing degree during the preparation of triturations with a mortar and pestle. We used powders with different distributions of particle sizes, i.e., powder that passed through 32-mesh but was trapped on a 42-mesh sieve (32/42-mesh powder), powder that passed through a 42 -mesh sieve but was trapped on a 60 -mesh sieve (42/60-mesh powder), powder that passed through a 60 -mesh sieve but was trapped on a 100-mesh sieve (60/100-mesh powder), and powder that passes through a 100-mesh sieve $(>100$ mesh powder). The mixing degree of colored powder and non-colored powder whose distribution of particle sizes was the same as that of the colored powder was excellent. The coefficient of variation $(\mathrm{CV})$ value of the mixing degree was $6.08 \%$ after 40 rotations when colored powder was mixed with non-colored powder that both passed through a 100 -mesh sieve. The CV value of the mixing degree was low in the case of mixing of colored and non-colored powders with different particle size distributions. After mixing, about $50 \%$ of $42 / 60$-mesh powder had become smaller particles, whereas the distribution of particle sizes was not influenced by the mixing of 60/100-mesh powder. It was suggested that the mixing degree is affected by distribution of particle sizes. It may be important to determine the mixing degrees for drugs with narrow therapeutic ranges.
\end{abstract}

Key words_- dispensation; trituration; particle size; granules

\section{INTRODUCTION}

The degree of mixing in trituration is affected by many factors, including the physical property and mixing ratio of the principal agents and diluents, the mixing conditions and the kind of blender used. Many studies using a V-type blender have been performed to determine the effects of various factors on mixing degree. ${ }^{1-4)}$ However, there has been little investigation of the effects of factors on the degree of mixing using a mortar and pestle, which are frequently used in hospitals and general pharmacies. It has been reported that a satisfactory degree of mixing cannot be achieved by using a mortar and pestle when powders and granules are mixed. ${ }^{5)}$ It has also been reported that the uniform mixing was achieved by using a mortar and pestle after $60-80$ rotations in the case of preparation of 5.0-30.0 g of powdered medicine when the gravity of the principal agent and that of diluents was similar. ${ }^{6-8)}$ In contrast to the fact that an excellent degree of mixing was achieved when powders with similar particle sizes were used, it has been

e-mail: igat-tky@umin.ac.jp reported that the mixing degree of powders with different particle sizes was unsatisfactory. Although the $10^{\text {th }}$ edition of the Japanese Guidelines for Dispensation indicates that medicines can be prepared by mixing powders and fine granules, there is no detailed information on the mixing degree of powders and fine granules.

In the present study, by using lactose colored with erythrocin, a food-coloring agent, we examined the effect of particle size on mixing degree during the preparation of triturations with a mortar and pestle, which are generally used for dispensation.

\section{MATERIALS AND METHODS}

Preparation of Lactose Lactose powder $(20 \mathrm{~g}$, lot number 099208, Yoshida Seiyaku Co., Ltd., Tokyo, Japan) was transferred to a glass mortar, and erythrocin $(2 \mathrm{~g}$, lot number GG41HH, Tokyo Kasei Kogyo Co., Ltd., Tokyo, Japan) dissolved in $5 \mathrm{ml}$ of distilled water was added to the mortar and mixed well. After addition of the remaining lactose powder $(78 \mathrm{~g})$, the solution was mixed until it was homogeneous and was then passed through a 32-mesh dispensing sieve (screen size of $500 \mu \mathrm{m}$ ). After dehydration 
at $60^{\circ} \mathrm{C}$ for $1 \mathrm{~h}$ using a circulating warm-air dehydrator, the resulting pellet was sieved through 100-mesh (screen size of $149 \mu \mathrm{m}$ ), 60-mesh (screen size of 250 $\mu \mathrm{m}$ ), 42-mesh (screen size of $350 \mu \mathrm{m}$ ) and 32-mesh (screen size of $500 \mu \mathrm{m}$ ) dispensing sieves. We could thus obtain powders with different distributions of particle sizes, i.e., powder that passed through a 32mesh sieve but was trapped on a 42-mesh sieve (32/ 42-mesh powder), powder that passed through a 42 mesh sieve but was trapped on a 60-mesh sieve (42/ 60-mesh powder), powder that passed through a $60-$ mesh sieve but was trapped on a 100-mesh sieve (60/ 100-mesh powder), and powder that passed through a 100 mesh sieve ( $>100$-mesh powder). Diluents were prepared from powdered lactose according to the method described above by using $5 \mathrm{ml}$ of distilled water free of erythrocin.

Methods of Mixing A china mortar with an outer diameter of $10 \mathrm{~cm}$ and depth of $5.5 \mathrm{~cm}$ (Nikkatou Seiyaku, Tokyo, Japan) was used. The mortar was held and rotated by the left hand, and the pestle was rotated spirally in the direction opposite to that of the rotation of the mortar. For the mixing, 10 clockwise rotations followed by 10 anticlockwise rotations were repeated three times, the mortar thus being rotated 60 times. Rotation was performed at a constant rate $(60$ rotations $/ 30 \mathrm{sec})$. In the present study, $10 \mathrm{~g}$ of colored lactose powders $(>100$-mesh, $60 / 100$ - and 42/60-mesh powders), and the same amount of diluent lactose $(>100-, 60 / 100-, 42 / 60$ and 32/42-mesh powders) were mixed in the mortar.

\section{Determination of Particle Size After mixing,} the distribution of particle sizes was determined by sieving the mixed powders through 32-, 42-, 60-, 80-, 100-, 150- and 200-mesh prescription sieves with screen sizes of $500,350,250,177,149,105$ and 74 $\mu \mathrm{m}$, respectively.

Estimation of Mixing Degree After mixing, 1.0 $\mathrm{g}$ specimens were randomly taken from three portions of the mixed powders to determine the concentration of erythrocin. Each specimen was dissolved in distilled water to obtain a solution of $10 \mathrm{ml}$. The absorption at the wavelength of $524 \mathrm{~nm}$ was determined by using a spectrophotometer (UV-160, Shimadzu Co., Ltd., Kyoto, Japan) .

Assuming that the contents of the major agents in the triturations were distributed according to the normal probability distribution, the coefficient of variation (CV) value was $6.08 \%$ under conditions in which $90 \%$ of the powder fraction contained $100 \pm 10$ $\%$ of the expected amount of the major agent. ${ }^{9}$ Therefore, the mixing degree was judged as excellent if the $\mathrm{CV}$ value of three determinations of erythrocin concentration in each preparation was less than 6.08 $\%$.

\section{RESULTS}

Effect of Particle Size on Mixing Degree At first, we examined the mixing degrees of colored lactose having particle size of $>100$-mesh with diluent lactose having particle sizes of $>100-, 60 / 100-, 42 /$ 60- and 32/42-mesh. The CV of the mixing degree became less than $6.08 \%$ after 40 rotations in the case of diluent lactose with particle size of $>100$-mesh (Fig. 1). In contrast, 60 rotations were required to achieve a $\mathrm{CV}$ value of less than $6.08 \%$ in the case of diluent lactose with particle sizes of 60/100-, 42/60and 32/42-mesh (Fig. 1).

It was also found that the $\mathrm{CV}$ value of the mixing degree of colored lactose having particle sizes of $60 /$ 100 and 42/60-mesh with diluent lactose having particle sizes of $>100-, 60 / 100-, 42 / 60-$ and 32/42-mesh became less than $6.08 \%$ after 60 rotations (Figs. 2 and 3).

Distribution of Particle Sizes after Mixing Distribution of particle sizes was examined after mixing colored lactose having particle size of $42 / 60$-mesh with diluent lactose having particle size of $42 / 60$ mesh. As shown in Table 1, after 100 rotations, $45 \%$ and $4 \%$ of the $42 / 60$-mesh particles had become 60 / 100- and 100/150-mesh particles, respectively, due to the grinding/destruction of particles in the mortar. In contrast, only $12 \%$ of the $60 / 100$-mesh particles had become 100/150-mesh particles after the mixing of 60 /100-mesh particles (Table 2).

\section{DISCUSSION}

One of the most important factors that determine the degree of mixing in trituration is particle sizes of principal drugs and diluents. In the present study, we determined mixing degrees by using colored lactose. The CV value of mixing degree became less than 6.08 $\%$ after 40 rotations when colored lactose was mixed with diluent lactose whose particle sizes were the same as that of the colored lactose, except in the case of 42/ 60 mesh powder. Moreover, the $\mathrm{CV}$ value of mixing became less than $6.08 \%$ after 40 rotations when colored lactose with particle size of $>100$-mesh was 


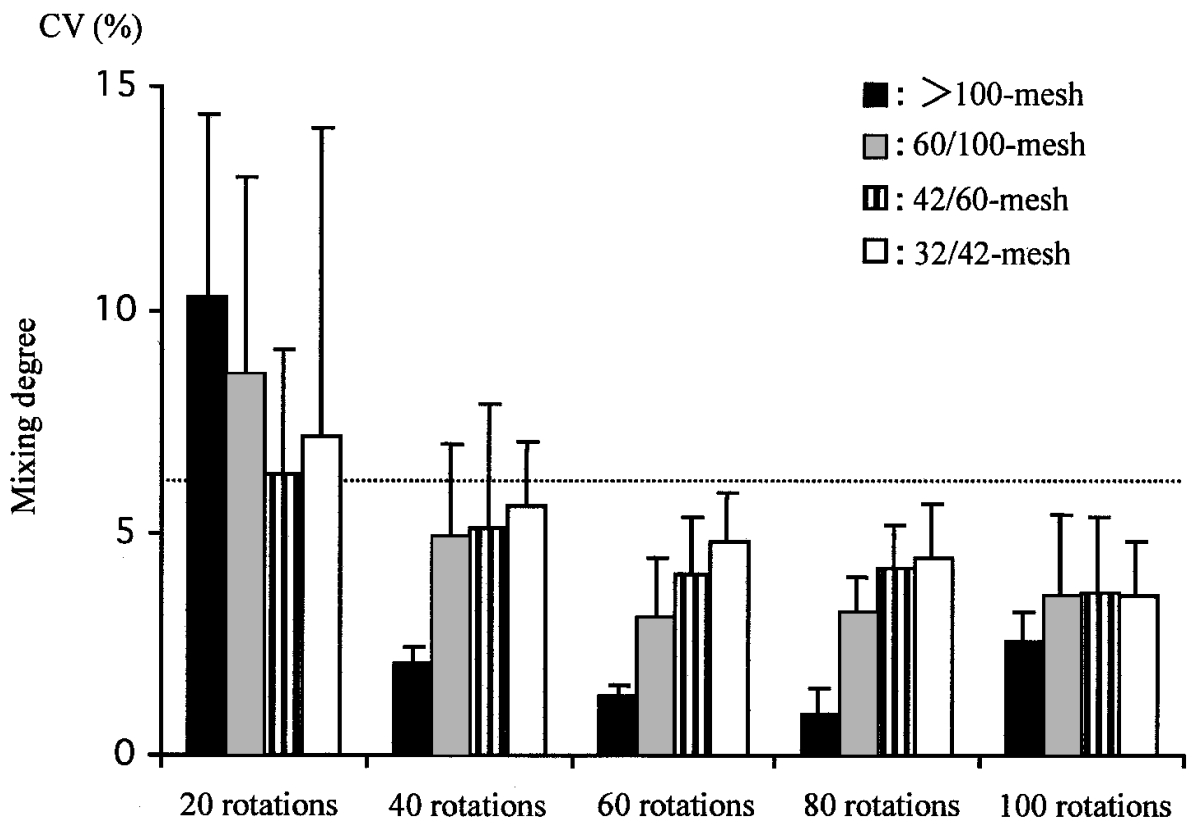

Fig. 1. Effect of Particle Size on Mixing Degree of $>100$-mesh Colored Lactose

Colored lactose having particle size of $>100$-mesh was mixed with the same amount of diluent lactose having particle sizes of $>100,60 / 100-, 42 / 60$ - and 32/42mesh. The mortar and pestle were rotated 20, 40, 60, 80 and 100 times. The results are given as CV value of the mixing degree. Each bar represents the mean \pm S.D. of three determinations.

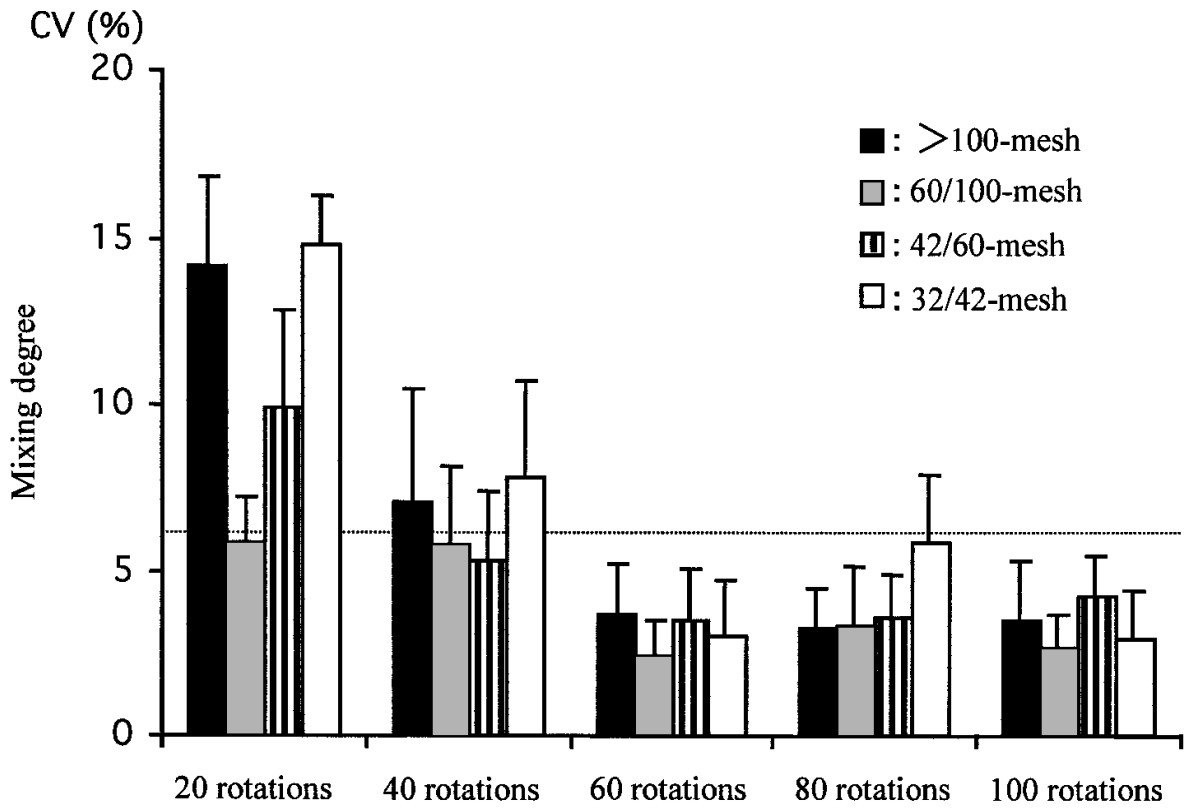

Fig. 2. Effect of Particle Size on Mixing Degree of 60/100-mesh Colored Lactose

Colored lactose having particle size of 60/100-mesh was mixed with the same amount of diluent lactose having particle sizes of $>100,60 / 100-$, 42/60- and 32/ 42-mesh. The mortar and pestle were rotated 20, 40, 60, 80 and 100 times. The results are given as CV of the mixing degree. Each bar represents the mean \pm S.D. of three determinations.

mixed with diluent lactose with particle size of $>100$ mesh, although we do not know the reason. It was also found that mixing degree was satisfactory when particle sizes of the colored and diluent lactose were the same than when they were different, especially for small colored lactose samples.

In addition, the distribution of particle sizes was examined after mixing lactose powders whose particle sizes were 42/60- and 60/100-mesh. After 100 rotations, approximately $50 \%$ of the $42 / 60$-mesh particles 


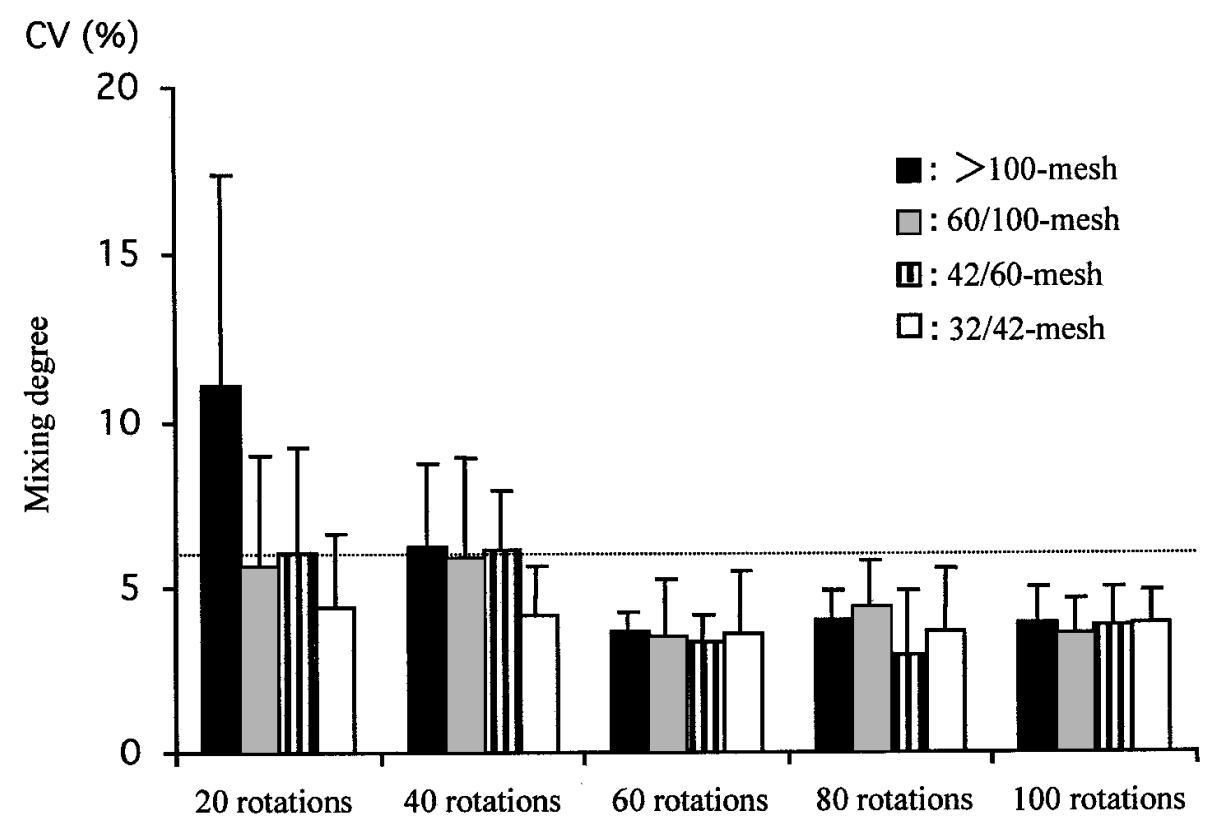

Fig. 3. Effect of Particle Size on Mixing Degree of 42/60-mesh Colored Lactose

Colored lactose having particle size of 42/60-mesh was mixed with the same amount of diluent lactose having particle sizes of $>100-, 60 / 100-$, 42/60- and 32/ 42-mesh. The mortar and pestle were rotated $20,40,60,80$ and 100 times. The results are given as CV of the mixing degree. Each bar represents the mean \pm S.D. of three determinations.

Table 1. Alterations in Particle Size Caused by Mixing of 42/ 60-Mesh Powders

\begin{tabular}{cc}
\hline \hline Particle size & Mean \pm S.D. (\%) \\
\hline $42 / 60$-mesh & $48.6 \pm 3.5$ \\
$60 / 100$-mesh & $45.3 \pm 4.2$ \\
100/150-mesh & $3.8 \pm 0.2$ \\
150/200-mesh & $0.9 \pm 0.3$ \\
$>200$-mesh & $1.4 \pm 0.5$ \\
\hline Total & 100 \\
\hline
\end{tabular}

Powder with particle size of 42/60-mesh was mixed in a mortar and a pestle by 100 rotations. After mixing, particle size was determined using dispensing sieves. Each value is the mean \pm S.D. of three determinations.

became smaller, whereas only $12 \%$ of the $60 / 100$ mesh particles had become 100/150-mes particles (Table 2). It was suggested that, in contrast to powders with small particle sizes, the mixing of large granules in the mortar results in an altered distribution of particle sizes. In preliminary experiments, we examined the distribution of particles by using a coated mortar, shaker and dispensing spoon. Forty five percent and $4 \%$ of the $42 / 60$-mesh particles became 60/100- and 100/150-mesh particles, respectively, when a coated mortar and shaker were used. These results are consistent with the results obtained by using a regular mortar. In addition, $38 \%$ of the $42 / 60$ mesh particles became 60/100-mesh particles after
Table 2. Alterations in Particle Size Caused by Mixing of 60/ 100-Mesh Powders

\begin{tabular}{cc}
\hline \hline Particle size & Mean \pm S.D. (\%) \\
\hline 60/100-mesh & $85.3 \pm 3.2$ \\
100/150-mesh & $12.3 \pm 1.8$ \\
150/200-mesh & $0.8 \pm 0.2$ \\
$>200$-mesh & $1.6 \pm 0.2$ \\
\hline Total & 100 \\
\hline Powders with particle size of 60/100-mesh was mixed in a mortara and \\
pestle by 100 rotations. After mixing, particle size was determined using \\
dispensing sieves. Each value is the mean \pm S.D. of three determinations.
\end{tabular}

mixing with a dispensing spoon. Taken together, the results indicate that alterations in the distribution of particle sizes occur after mixing using a regular or coated mortar, a shaker, and a dispensing spoon instead of a pestle.

Concerning the selection of diluents for preparation of triturations of granules, Aoki et al. ${ }^{7)}$ and Kobo et al. ${ }^{10)}$ reported that the diluent powder that passed through a 35-mesh sieve and powder that passed through a 50-mesh sieve but was trapped by 100-mesh sieve were suitable. Kozatani et al ${ }^{9)}$ reported that the diluent powder that passed through a 36mesh sieve but was trapped by 65 -mesh sieve was suitable considering convenience in handling (such as 
mixing degree, repose angle and elusion coefficient). However, the distributions of particle sizes of powders and fine granules are different from each other, which is based on the definition of fine granules in the Japanese Pharmacopeia. Fine granules are defined in the $9^{\text {th }}$ and $10^{\text {th }}$ editions of the Japanese Pharmacopeia as particles for which less than $10 \%$ pass through a 200-mesh sieve. In contrast according to the Requirements for Antibiotic Products of Japan, 1993, fine granules are defined as particles for which more than $95 \%$ pass through a 32 -mesh sieve. Consequently, the distribution of particle sizes of commercially available fine granules which consist of principal drugs is different from each other depending on the products. For example, approximately 10\%, 18 $\%, 37 \%$ and $34 \%$ of commercially available Aleviatin fine granules are 32/42-, 42/60-, 60/100- and $>100-$ mesh particles, respectively, whereas approximately $62 \%$ of commercially available Serenace fine granules are trapped on a 48 mesh sieve. It is therefore possible that the degree of mixing of fine granules with powders is affected not only by the distribution of particle sizes but also by the degree of hardness of fine granules, since fine granules with a low degree of hardness may become smaller during mixing.

From these findings, it was suggested that the mixing degree is affected by the distribution of particle sizes but that 60 rotations may result in excellent mixing for particles of less than 32/42-mesh in size. For preparation of triturations from fine granules with relatively large diameters, the degree of hardness of the fine granules may affect the mixing degree, since fine granules with a low degree of hardness may become smaller during mixing.

\section{REFERENCES}

1) Okada J., Matsuda Y., Morita O., Wada Y., Onishi H., Yakugaku Zasshi, 88, 827-831 (1968).

2) Okada J., Matsuda Y., Fukumori Y., Yakugaku Zasshi, 92, 270-274 (1972).

3) Awata E., Ohtsuka K., Morioka T., Ikegami Y., Yakuzaigaku, 19, 141-144 (1959).

4) Ueno T., Saito M., Suzuki K., Yakuzaigaku, 19, 244-247 (1959).

5) Yamana T., Minakami Y., Ichimura F., Isemi Y., Yakuzaigaku, 32, 21-26 (1972).

6) Sakurai K., Fujii S., Ito S., Yakuzaigaku, 16, 7-12 (1956).

7) Aoki D., Fukuda T., Ueda S., Mochiduki A., Yakuzaigaku, 27, 106-108 (1967).

8) Nakamura H., Higo K., Suzuki A., Fujinuma Y., Tanaka Y., Ohtani M., Kotaki H., Iga T., Jpn. J. Hosp. Pharm., 23, 306-311 (1997).

9) Kozatani J., Kitaura T., Ashida K., Yakuzaigaku, 29, 53-56 (1969).

10) Kobo B., Inoue S., Jpn. J. Hosp. Pharm., 3, 215-219 (1978). 